\title{
shinyCurves, a shiny web application to analyse multisource qPCR amplification data: a COVID-19 case study
}

\author{
S. Olaechea-Lázaro ${ }^{1}$, I. García-Santisteban ${ }^{1,2}$, J. R. Pineda ${ }^{3,4}$, I. Badiola ${ }^{4}$, S. Alonso ${ }^{1}$, Jose Ramon Bilbao ${ }^{1,2^{*+}}$ and \\ Nora Fernandez-Jimenez ${ }^{1,2^{*}+}$ (D)
}

*Correspondence: joseramon.bilbao@ehu.eus; nora.fernandez@ehu.eus †Jose Ramon Bilbao and Nora Fernandez-Jimenez have contributed equally to this work

${ }^{1}$ Department of Genetics, Physical Anthropology and Animal Physiology, Faculty of Medicine and Nursing, University of the Basque Country (UPV/ EHU), Barrio Sarriena s/n, 48940 Leioa, Spain Full list of author information is available at the end of the article

\begin{abstract}
Background: Quantitative, reverse transcription PCR ( $q R T-P C R$ ) is currently the goldstandard for SARS-CoV-2 detection and it is also used for detection of other virus. Manual data analysis of a small number of qRT-PCR plates per day is a relatively simple task, but automated, integrative strategies are needed if a laboratory is dealing with hundreds of plates per day, as is being the case in the COVID-19 pandemic.

Results: Here we present shinyCurves, an online shiny-based, free software to analyze qRT-PCR amplification data from multi-plate and multi-platform formats. Our shiny application does not require any programming experience and is able to call samples Positive, Negative or Undetermined for viral infection according to a number of userdefined settings, apart from providing a complete set of melting and amplification curve plots for the visual inspection of results.
\end{abstract}

Conclusions: shinyCurves is a flexible, integrative and user-friendly software that speeds-up the analysis of massive qRT-PCR data from different sources, with the possibility of automatically producing and evaluating melting and amplification curve plots.

Keywords: Diagnosis, qRT-PCR, Melting and amplification curves, COVID-19, Data analysis, Medical informatics, Virology, Shiny application

\section{Background}

Quantitative, reverse transcription polymerase chain reaction (qRT-PCR) is a widely used technique for the detection and quantification of mRNA. Currently, with the spread of COVID-19, and despite the development of a number of interesting imaging and artificial intelligence-based diagnostic procedures [1, 2], qRT-PCR is still considered the gold-standard tool for SARS-CoV-2 detection by the World Health Organization (WHO) [3]. Depending on the amount of viral mRNA detected by the qRT-PCR system, a sample is assigned as Positive, Negative or Undetermined. Most healthcare providers rely on automated detection procedures that make use of proprietary reagents and software and offer limited options for parameter set-up, with special emphasis on author(s) and the source, provide a link to the Creative Commons licence, and indicate if changes were made. The images or other third party material in this article are included in the article's Creative Commons licence, unless indicated otherwise in a credit line to the material. If material is not included in the article's Creative Commons licence and your intended use is not permitted by statutory regulation or exceeds the permitted use, you will need to obtain permission directly from the copyright holder. To view a copy of this licence, visit http:// creativecommons.org/licenses/by/4.0/. The Creative Commons Public Domain Dedication waiver (http://creativecommons.org/publi cdomain/zero/1.0/) applies to the data made available in this article, unless otherwise stated in a credit line to the data. 
those recommended by WHO (CFX Manager 3.1 from BioRad) and by the FDA (Food and Drug Administration) (the SDS 1.4 software from Applied Biosystems).

In this context, research laboratories worldwide are developing alternative diagnostic protocols that do not depend on commercial kits and their accompanying software. Thus, researchers may choose from a wide variety of quantitative gene expression reagents (one- vs. two-step qRT-PCR or fluorescent probes $v s$. intercalating dyes) and adapt protocols to the real-time amplification systems available [4]. In addition to the need for independent experimental protocols, there is also a need for an open-source tool that can rapidly analyze qRT-PCR data irrespective of the protocol or equipment used. Additionally, in order to discard samples with unspecific amplification products, a software for accurate qRT-PCR analysis should allow the inspection of amplification and melting curves.

Finally, the increase in the number of worldwide infections, driven by fast-spreading variants that are especially relevant in low-income countries, makes qRT-PCR the first choice for SARS-Cov-2 detection. There is a need for open-source tools for qRT-PCR data analysis that are easily customizable to the experimental set-up in each setting, without the prohibitive cost of proprietary licenses. Furthermore, the high risk in dense populations together with the close contact of SARS-CoV-2 reservoir in host's animals makes the qRT-PCR detection an urgent need with strong public health implications [5, 6].

In this context, and taking into account both WHO recommendations and the new challenges of the global COVID-19 pandemic, the objective of our work was to develop a flexible, fast and non-proprietary software for massive qRT-PCR data analysis. Herein, we present shinyCurves, a Shiny-based, user-friendly, flexible, integrative, non-proprietary and free application that is able to:

1 Process qRT-PCR raw amplification data obtained with either fluorescent probes or intercalating dyes, from different plate formats and qRT-PCR systems, including those recommended by the main public health agencies;

2 Establish the settings that will classify samples into three categories (Positive, Negative or Undetermined), and to include both a range of optional experimental controls, and the possibility of using serial dilutions of viral RNA/DNA;

3 Plot both amplification and melting curves, providing additional quality control of the specificity of the amplification and offering the possibility of visually inspecting the results obtained.

A COVID-19 toy dataset is also provided as a practical example (see Additional file 1).

\section{Implementation}

shinyCurves is designed for users with limited or no programming experience who wish to analyze qRT-PCR data in a simple and efficient manner. The application was completely written using R [7] in combination with the 'shiny' package [8], and can be found in the shinyapps.io repository as a web application (https://biosol.shinyapps.io/shiny curves/). The source code can be freely downloaded from the GitHub repository https:// github.com/biosol/shinyCurves. Analysis tables are processed by 'data.table' [9] and 
'dplyr' [10] due to their high efficiency, and plots are generated using 'ggplot2' [11] and 'plotly' [12]. Specifically, to plot melting curves the 'qpcR' R package [13] is used.

Raw data can be uploaded directly to the application in different file formats, including csv, xlsx or xls files generated in the two most widely used qPCR systems (i.e. BioRad and Applied Biosystems platforms), as pointed out in Fig. 1. Once the upload is complete, the intercalating dye pipeline will start by plotting a melting curve per reaction, in order to discard those that show unspecific amplification products, before sample classification into the Positive, Negative and Undetermined groups. In the case of the fluorescent probe pipeline, it will first perform the classification of the samples or the calling analysis, and then plot the amplification curves for visual inspection of the results.

Finally, it is worth to mention that shinyCurves allows for the use of sample duplicates and is also independent from the plate format, i.e. 96- or 364-well plates. The inclusion of experimental controls and serial dilutions of viral DNA is optional, but if included, must follow specific formats, as described in the provided Manual (see Additional file 2).

\section{Results}

To illustrate the shinyCurves pipeline, data sets generated by the COVID-19 Basque Inter-Institutional Group (coBIG) in both fluorescent probe and intercalating dye experiments, and run on the BioRad CFX and Applied Biosystems Quant Studio systems are provided [4]. The genes chosen for these analyses are N1, RdRp and RNAseP (human




genomic control) in the fluorescent probe assay, and $N, S, R d R p$ and $H 30$ (endogenous control) in the intercalating dye assay. These genes are included in the COVID-19 diagnostic panel described by the US Centers for Disease Control and Prevention (CDC, https://www.cdc.gov/).

\section{Melting curves (in the intercalating dye analysis)}

In the dye experiments, shinyCurves allows users to plot melting curves to exclude from the final calling those samples lacking reliable and unique melting temperature (Tm) peaks. These plots are generated using the meltcurve function from the 'qpcR' package in R [13]. As a result, users can download a table including only those plate wells that contain samples with unique Tm peaks that meet the established criteria, together with the Tm value and plot assigned to each well. This table must be included in the calling analysis as a prior filtering step (ID_well file).

\section{Calling analysis}

After uploading the input data, users are allowed to fine-tune a series of parameters that will be used to classify samples as Positive, Negative or Undetermined. In general, two types of calling criteria are allowed, namely, the Ct value of the analyzed viral gene(s) (compulsory) and the estimated viral RNA copy number (optional). A human endogenous control is included to make sure that the nucleic acid extraction worked. Adjustable parameters include, among others: use of a viral DNA standard curve (yes/no), use of duplicates (yes/no) and number of "positive" viral genes to consider a sample Positive. Calling results are presented in a downloadable table. For more details on the calling criteria, see Additional file 3, where the algorithm of the full Calling Analysis has been described in detail.

\section{Amplification curves (in the fluorescent probe analysis)}

After performing the calling analysis in probe experiments, shinyCurves allows to plot General Amplification Curves including all the samples, as well as individual Amplification Curves of samples classified as Undetermined. Each Undetermined sample is plotted independently, together with those with a final calling. Upon visual inspection of Undetermined sample curves, the user can decide whether these fit a sigmoidal distribution, and therefore represent a specific amplification, or not.

\section{Conclusions}

To our knowledge, this is the first tool designed to automatize the calling of clinical samples containing pathogen nucleic acids through qRT-PCR, and that is completely flexible as regards the user's requirements and experimental settings. In fact, several open-access software packages and tools for the analysis of qPCR data already exist (see review by [14]). However, some of them either have been discontinued (CopyCaller) or are no longer maintained [15] or need a subscription or license (Cy0 Method, https:// www.cy0method.org/, [16]). Moreover, the alternatives for drawing melting curves other than proprietary software are very limited. As far as we know, the qpcR R package is the only available free package, but no graphical user interface is provided [17]. 
In summary, shinyCurves is a user-friendly application that analyzes and allows the visualization of qRT-PCR data coming from different amplification methods and platforms. It is easily accessible for any user profile, as no programming skills are required. shinyCurves is set up automatically in the shinyapps.io server, making basic Internet connection its only requirement. Its minimal requirements make it a ready-to-use tool applicable to the clinical routine. Therefore, we conclude that it is a significant improvement in analytical capacity, speed and reproducibility, which are key factors in pathogen detection analyses, especially in COVID-19 times.

\section{Abbreviations}

qRT-PCR: reverse transcription polymerase chain reaction; WHO: World Health Organization; FDA: Food and Drug Agency; coBIG: COVID-19 Basque Inter-Institutional Group; CDC: US Centers for Disease Control and Prevention; Tm: melting temperature.

\section{Supplementary Information}

The online version contains supplementary material available at https://doi.org/10.1186/s12859-021-04392-1.

Additional file 1. COVID-19 toy dataset. A COVID-19 toy dataset containing example files coming from (a) a fluorescent probe analysis (Applied Biosystems Quant Studio) and (b) an intercalating dye analysis (BioRad CFX).

Additional file 2. shinyCurves manual. Contains a precise description of all the options implemented in shinyCurves, as well as a tutorial describing the analysis of the two COVID-19 datasets provided.

Additional file 3. Result assignment criteria in the Calling Analysis. In the upper section, samples are assigned a result based on their viral gene Ct values (compulsory). First, when duplicates are included in the analysis, mean $\mathrm{Ct}$ is calculated and samples with divergent duplicates are marked as Undetermined. Then, in (2), mean (samples with duplicates) or individual (samples with no duplicates) Ct values are compared against the maximum Ct value (MaxCt) selected by the user and each sample is assigned a result (Positive, Negative, Check Copy Number). In (3), the total number of Positive, Negative or Check Copy Number assignments is compared to the number of Positive genes necessary to assign a sample as Positive (inputted by the user) and the sample is assigned a final result (Positive, Negative, Undetermined or Check Copy Number). If the user does not want to consider the copy number as a result assignation criterion, the analysis is over. Otherwise, the analysis continues in the blue section in which samples are assigned a result based on their estimated viral DNA copy number (optional). In (4a), the estimated copy number is compared to the minimum copy number inputted by the user and the gene is assigned as Positive or Undetermined. Finally, in (4b), similarly to (3), the gene result assignment is compared to the number of Positive genes necessary to assign a sample as Positive and the sample is assigned as either Positive or Undetermined.

Acknowledgements

We would like to thank coBIG \& "Acción especial COVID-19" participating scientists and voluntaries for the COVID-19 samples and material.

\section{Authors' contributions}

JRB, NFJ and IGS planned all statistical analyses. SOL and NFJ programmed the tool and drafted the manuscript. SOL, IGS, $J R P, I B, S A, J R B$ and NFJ tested the application at different stages of development and critically reviewed the manuscript. All authors contributed critically to the development of the tool. All authors read and approved the final manuscript.

\section{Funding}

This project was supported by funding from the UPV/EHU (Acción Especial "Desarrollo e implementación del test de diagnóstico para COVID-19"). The funding body did not play any roles in the study design; nor in the data collection, analysis and interpretation, or in the writing of the paper.

\section{Availability of data and materials}

Project name: shinyCurves. Project home page: https://biosol.shinyapps.io/shinycurves/. Operating system(s): Platform independent. Programming language: R. Other requirements: basic Internet connection. License: CC BY-NC-SA 4.0. Any restrictions to use by non-academics: CC BY-NC-SA 4.0. The toy dataset analyzed in this study can be downloaded from https://biosol.shinyapps.io/shinycurves/.

\section{Declarations}

Ethics approval and consent to participate

The study was conducted at the University of the Basque Country (UPV/EHU, Leioa, Spain) in collaboration with the Cruces University Hospital after approval by the UPV/EHU Ethics Committee (Project 2020/059, CEIAB M30/2020/074). 


\section{Consent for publication \\ Not applicable.}

\section{Competing interests}

The authors declare that they have no competing interests.

\section{Author details}

${ }^{1}$ Department of Genetics, Physical Anthropology and Animal Physiology, Faculty of Medicine and Nursing, University of the Basque Country (UPV/EHU), Barrio Sarriena s/n, 48940 Leioa, Spain. ${ }^{2}$ Biocruces-Bizkaia Health Research Institute, Plaza de Cruces, 48903 Barakaldo, Spain. ${ }^{3}$ Achucarro Basque Center for Neuroscience, Barrio Sarriena s/n, 48940 Leioa, Spain. ${ }^{4}$ Department of Cell Biology and Histology, Faculty of Medicine and Nursing, University of the Basque Country (UPV/EHU), Barrio Sarriena s/n, 48940 Leioa, Spain.

Received: 13 November 2020 Accepted: 22 September 2021

Published online: 03 October 2021

\section{References}

1. Saha P, et al. EMCNet: automated COVID-19 diagnosis from X-ray images using convolutional neural network and ensemble of machine learning classifiers. Inform Med. 2021;22:100505.

2. Islam MM, et al. A review on deep learning techniques for the diagnosis of novel coronavirus (COVID-19). IEEE Access. 2021:9:30551-72.

3. World Health Organization. Diagnostic testing for SARS-CoV-2: interim guidance. World Health Organization. 11 th Sept 2020. https://apps.who.int/iris/handle/10665/334254. License: CC BY-NC-SA 3.0 IGO. Accessed Sept 2020.

4. Guruceaga $X$, et al. Fast SARS-CoV-2 detection protocol based on RNA precipitation and RT-qPCR in nasopharyngeal swab samples. MedRxiv. 2020. https://doi.org/10.1101/2020.04.26.20081307.

5. Sharun K, Dhama K, Pawde AM, Gortázar C, Tiwari R, Bonilla-Aldana DK, et al. SARS-CoV-2 in animals: potential for unknown reservoir hosts and public health implications. Vet Q. 2021;41:1-31.

6. Sharun K, Tiwari R, Natesan S, Dhama K. SARS-CoV-2 infection in farmed minks, associated zoonotic concerns, and importance of the One Health approach during the ongoing COVID-19 pandemic. Vet Q. 2021;41:50-60.

7. R Core Team. R: a language and environment for statistical computing. Vienna: R Foundation for Statistical Computing; 2020.

8. Chang W, et al. shiny: web application framework for R. 2000. R package version 1.4.0.2. https://CRAN.R-project.org/ package=shiny. Accessed Sept 2020.

9. Dowle M, Srinivasan A. data.table: extension of 'data.frame'. 2019. R package version 1.12.8. https://CRAN.R-project. org/package=data.table. Accessed Sept 2020.

10. Wickham H, François R, Henry L, Müller K. dplyr: a grammar of data manipulation. 2020. R package version 1.0.0. https://CRAN.R-project.org/package=dplyr. Accessed Sept 2020.

11. Wickham H. ggplot2: elegant graphics for data analysis. New York: Springer; 2016.

12. Sievert C. Interactive web-based data visualization with R, plotly, and shiny. Boca Raton: Chapman and Hall; 2020.

13. Spiess A-N. qpcR: modelling and analysis of real-time PCR data. 2018. R package version 1.4-1. https://CRAN.R-proje ct.org/package=qpcR. Accessed Sept 2020.

14. Pabinger $S$, Rödiger $S$, Kriegner A, Vierlinger $K$, Weinhäusel A. A survey of tools for the analysis of quantitative $P C R$ (qPCR) data. Biomol Detect Quantif. 2014;1:23-33.

15. Blom J, Rückert C, Kalinowski J, Goesmann A. CAmpER - a software for the calculation of amplification efficiencies for real-time PCR-experiments. 2007. http://camper.cebitec.uni-bielefeld.de/. Accessed Sept 2020.

16. Guescini M, Sisti D, Rocchi MBL, Panebianco R, Tibollo P, Stocchi V. Accurate and precise DNA quantification in the presence of different amplification efficiencies using an improved Cy0 method. PLoS ONE. 2013;8:e68481.

17. Ritz C, Spiess A-N. qpcR: an R package for sigmoidal model selection in quantitative real-time polymerase chain reaction analysis. Bioinformatics. 2008;24:1549-51.

\section{Publisher's Note}

Springer Nature remains neutral with regard to jurisdictional claims in published maps and institutional affiliations. 